\title{
L'élection présidentielle de 2015 en Côte d'Ivoire : une victoire pour Ouattara, mais pas un plébiscite
}

Christian Bouquet and Irène Kassi-Djodjo

\section{(2) OpenEdition}

\section{Journals}

Electronic version

URL: http://journals.openedition.org/echogeo/14454

DOI: 10.4000/echogeo.14454

ISSN: 1963-1197

\section{Publisher}

Pôle de recherche pour l'organisation et la diffusion de l'information géographique (CNRS UMR 8586)

\section{Electronic reference}

Christian Bouquet and Irène Kassi-Djodjo, «L'élection présidentielle de 2015 en Côte d'Ivoire : une victoire pour Ouattara, mais pas un plébiscite », EchoGéo [Online], Sur le Vif, Online since 15 February 2016, connection on 19 April 2019. URL : http://journals.openedition.org/echogeo/14454 ; DOI : 10.4000/echogeo.14454

This text was automatically generated on 19 April 2019.

\section{cc) (†)}

EchoGéo est mis à disposition selon les termes de la licence Creative Commons Attribution - Pas d'Utilisation Commerciale - Pas de Modification 4.0 International 


\title{
L'élection présidentielle de 2015 en Côte d'Ivoire : une victoire pour Ouattara, mais pas un plébiscite
}

\author{
Christian Bouquet and Irène Kassi-Djodjo
}

\section{Introduction : une élection pour tourner la page de 2010}

1 À la veille de l'élection présidentielle du 25 octobre 2015, on comptait encore 5500 casques bleus de l'ONUCI en Côte d'Ivoire. Le contingent, déployé en 2004, avait sensiblement diminué depuis le pic de 2010 (8500 hommes), mais la présence onusienne avait été prorogée jusqu'en 2016, signe que subsistait encore une certaine inquiétude sur la sécurité et la paix dans l'ensemble du territoire. Le scrutin était donc attendu avec inquiétude, à la fois par les électeurs ivoiriens et par la communauté internationale.

Chacun gardait d'ailleurs en mémoire la rapidité avec laquelle le pays avait basculé dans un début de guerre civile lors du scrutin précédent, dès le lendemain du second tour (28 novembre 2010), lorsque le camp du président sortant Laurent Gbagbo avait choisi de contester les résultats communiqués par la Commission électorale indépendante qui donnaient Alassane Ouattara vainqueur avec $54,1 \%$ des suffrages. Aux ordres, le Conseil constitutionnel avait inversé les chiffres, et il avait fallu que le représentant spécial du Secrétaire général des Nations unies use du mandat qui lui avait été confié pour confirmer, grâce à un recomptage strict, le verdict livré par la Commission électorale indépendante (Choi, 2015).

Il s'en était suivi près de cinq mois d'affrontements meurtriers, faisant officiellement plus de 3000 morts et autant de « disparus ", jusqu'à ce que les troupes de l'ONU appuyées par la force française d'intervention spéciale Licorne contraignent l'ex-président Laurent Gbagbo à se rendre, le 11 avril 2011 (Notin, 2013), mettant ainsi fin à une bicéphalie inédite puisque chacun des deux candidats avait prêté serment devant la nation et se considérait comme chef de l'État. 
Cette crise postélectorale n'avait fait qu'aggraver un clivage qui frappait la Côte d'Ivoire depuis la fin des années 1990, lorsque l'ex-président Henri Konan Bédié avait laissé s'insinuer dans les esprits la théorie de l' " ivoirité » selon laquelle la nationalité devait reposer sur des critères d'autochtonie que seules les populations du sud semblaient pouvoir remplir. Peu après avoir accédé au pouvoir par le putsch militaire du 23 décembre 1999, le général Robert Gueï avait proposé une nouvelle Constitution qui rendait inéligible tout candidat ne pouvant justifier d'une double ascendance ivoirienne.

Alassane Ouattara n'ayant pu démontrer que son père ET sa mère étaient nés ivoiriens, il fut écarté du scrutin présidentiel d'octobre 2000, remporté par Laurent Gbagbo dans des conditions douteuses, et c'est à partir de cette exclusion que la Côte d'Ivoire sombra dans dix années de crise, dont cinq ans de partition du pays (2002-2007). Prévue pour 2005, l'élection présidentielle fut sans cesse reportée jusqu'en octobre 2010, et, contrairement à ce que l'on pouvait espérer, elle déboucha sur une exacerbation des oppositions entre les deux camps politiques ${ }^{1}$ mais aussi entre les communautés ethno régionales (Bouquet, 2011).

6 Lors de sa seconde prestation de serment, le 21 mai 2011, Alassane Ouattara démarrait donc un mandat sous haute tension, et sa marge de manœuvre allait être étroite.

\section{Cinq années de « retour à la normale »}

7 La chute de Laurent Gbagbo n'avait pas réglé tous les problèmes, notamment dans le domaine de la sécurité du pays, car en dix années de crise plusieurs milliers de civils avaient été - imprudemment - armés. Toutes les sorties de crise en Afrique butent généralement sur ce passage obligé qu'est le désarmement ${ }^{2}$, et la Côte d'Ivoire n'y a pas échappé. Nommés miliciens dans le camp de l'ex-chef de l'État et combattants dans celui du nouveau président, ils étaient environ 75000 à détenir au moins une arme et prêts à continuer à en vivre, faute d'autre solution.

8 Plusieurs opérations de DDR (Désarmement, Démobilisation, Réinsertion) avaient été entreprises sans succès depuis 2003. Le chantier lancé en 2011, doté d'un budget confortable (150 millions de dollars), s'est achevé en 2015 sur un bilan positif : 69506 exsupplétifs (dont 6105 femmes) ont été désarmés et réinsérés professionnellement, après avoir suivi un "stage de resocialisation" au cours duquel ils ont réappris à vivre ensemble. Certes le compte n'y est pas tout à fait puisqu'il n'a été récupéré que 39279 armes, mais chacun s'accorde à considérer que le DDR en Côte d'Ivoire pourra servir d'exemple pour les autres pays.

Parallèlement à la sécurisation du pays, la « réconciliation nationale » était la priorité du nouveau pouvoir, en même temps qu'une nécessité vitale pour les Ivoiriens et une exigence incontournable des chancelleries étrangères. Or l'exercice démarra sur un malentendu inhérent à une mauvaise compréhension du concept démocratique: les partisans de Laurent Gbagbo estimaient qu'il fallait réconcilier les $46 \%$ d'électeurs qui avaient voté pour lui avec les $54 \%$ d'électeurs d'Alassane Ouattara. Dans de telles conditions, la CDVR (Commission «Dialogue Vérité et Réconciliation») mise en place le 13 juillet 2011 sous la présidence de Charles Konan Banny allait avoir du mal à faire avancer l'idée, a fortiori parce que la Justice n'avait pas été incluse dans sa feuille de route. La CDVR, dont le rapport final n'a pas été rendu public, et dont le président a basculé 
dans le jeu politique en présentant sa candidature à l'élection présidentielle, a donc été un échec.

Entretemps, le relais avait été assuré par deux instances censées être plus efficaces: le PNCS (Programme national de Cohésion sociale) et surtout la CONARIV (Commission nationale de Réconciliation et d'Indemnisation des Victimes), chargée de distribuer 17 millions de dollars aux 158238 victimes recensées. Il semblerait que cette méthode soit de nature à réduire plus rapidement les cicatrices, mais elle n'est pas sans risques.

En outre, elle ne concerne pas ce qui restera encore longtemps la pierre d'achoppement de la réconciliation nationale, c'est-à-dire le sort de Laurent Gbagbo. Transféré à la CPI dès le 29 novembre 2011, rejoint à la Haye par son ancien ministre de la Jeunesse Charles Blé Goudé le 22 mars 2014, il a longtemps attendu son procès, qui a démarré en janvier 2016. Pour l'un et l'autre les chefs d'inculpation sont lourds ${ }^{3}$, mais cela n'empêche pas leurs partisans d'exiger leur libération (ainsi que celle de Simone Gbagbo ${ }^{4}$ ) comme préalable à toute reprise du dialogue politique.

Il n'est donc pas certain que la réconciliation de la nation soit réellement achevée. Au 15 avril 2015, le HCR recensait encore 59000 réfugiés ivoiriens à l'extérieur du pays, dont 38000 au Liberia, 11500 au Ghana et 6500 en Guinée. À la même date, on estimait à 240000 le nombre de ceux qui étaient rentrés en Côte d'Ivoire, soit environ 80 \% de ceux qui étaient partis.

13 Aussi est-ce dans un contexte encore fragile que le nouveau pouvoir d'Alassane Ouattara a organisé les élections intermédiaires, que les pro-Gbagbo ont d'ailleurs systématiquement boycottées. Dès le mois de décembre 2011 eurent lieu les élections législatives, marquées par une forte abstention (participation de 36,5\%), par de nombreux cas de dissidence, et par une large victoire du RDR, le parti d'Alassane Ouattara, qui obtenait la majorité absolue (138 sièges sur 255). Le PDCI se contentait de 86 sièges (Bouquet, Kassi-Djodjo, 2012) $)^{5}$.

14 Puis, en avril 2013, sans changer ni la liste électorale ni la Commission électorale indépendante, furent organisées simultanément les élections régionales et municipales. La participation fut un peu meilleure ( $44,3 \%$ aux élections régionales) et les listes d'union RHDP plus nombreuses mais, en l'absence de candidatures d'opposition, il était difficile d'en tirer des enseignements, sinon que la vie politique respectait le calendrier normal des scrutins intermédiaires et locaux (Bouquet, Kassi-Djodjo, 2014-1).

Parallèlement, l'économie du pays avait retrouvé toutes ses couleurs, avec un taux de croissance frisant les deux chiffres, tandis que des chantiers spectaculaires soulignaient la volonté du président Ouattara de régler les problèmes par le développement. L'ouverture du troisième pont sur la lagune Ébrié à Abidjan symbolisait la dynamique d'ensemble, et les investisseurs revenaient nombreux, ce qui fit dire dès janvier 2013 à Christine Lagarde, directrice générale du FMI, que l'on assistait au second miracle ivoirien ${ }^{6}$.

Mais le seul véritable indicateur d'un "retour à la normale " restait l'organisation de l'élection présidentielle de 2015. En voulant respecter scrupuleusement les textes constitutionnels, Alassane Ouattara en avait fixé la date au 25 octobre. Ce calendrier allait être difficile à tenir ${ }^{7}$.

D’abord parce que - signe récurrent en Afrique de démocraties immatures - il fallait réformer la Commission électorale indépendante (CEI), dont la composition reposait sur les équilibres politiques de 2003. Bien qu'affaiblie, l'opposition retarda de plusieurs mois son accord sur la nouvelle formule, dont la principale innovation était l'entrée de 
4 membres de la « société civile " parmi les 17 commissaires. Il fallait ensuite actualiser le code électoral qui avait quinze ans d'âge, et les navettes entre la CEI et le gouvernement prirent beaucoup de temps. Il ne restait donc que quelques mois à cette même CEI pour mettre à jour la liste électorale, sur laquelle le nombre d'électeurs n'avait pratiquement pas bougé depuis l'élection de 2000. Ce fut son échec le plus patent, puisqu'on attendait l'enrôlement des 9020891 Ivoiriens âgés de plus de 18 ans estimés par le Recensement de décembre $2014^{8}$, alors que la liste définitive s'est arrêtée à 6301189 inscrits (contre 5780804 en 2010). Pour un pays de 23 millions d'habitants, et même en décomptant les 25 $\%$ d'étrangers ${ }^{9}$, c'est très peu.

Le défi de l'organisation logistique incombait également à la CEI, dont on se souvenait qu'elle avait été à la peine en 2010, et qu'elle n'y serait pas parvenue sans l'appui massif de l'ONUCI. Or le mandat de celle-ci ne prévoyait plus un tel soutien, et l'approche de la date fatidique suscitait ici et là quelques légitimes inquiétudes.

\section{L'élection présidentielle de 2015}

19 Cependant, les opinions publiques nationale et internationale étaient davantage focalisées sur un concept qui fait florès à la moindre occasion : l'inclusivité, c'est-à-dire la possibilité pour toutes les sensibilités politiques de participer au scrutin. De fait, pour réellement «tourner la page » des années de crise, il fallait que l'élection présidentielle de 2015 soit exemplaire.

20 À l'instar du paysage urbain d'Abidjan, devenu méconnaissable à force de grands travaux, le paysage politique ivoirien avait sensiblement changé. Du côté de la majorité présidentielle, qui regroupait les cinq partis membres du RHDP (Rassemblement des Houphouëtistes pour la Paix et le Développement), l'unité semblait avoir perduré. Le 17 septembre 2014, Henri Konan Bédié, leader du PDCI, avait lancé l'Appel de Daoukro par lequel il demandait à ses électeurs de voter dès le premier tour pour Alassane Ouattara, qui devenait donc le candidat unique du RHDP. Dès lors, la partie paraissait gagnée d'avance pour le président sortant, même si une petite frange du PDCI allait refuser de s'aligner sur l'Appel, notamment deux caciques de l'ancien parti unique (Charles Konan Banny et Amara Essy) et un « jeune $»^{10}$ député frondeur (Konan Kouadio Bertin, dit KKB).

21 En face, le Front populaire ivoirien (FPI, parti de Laurent Gbabgo) avait fini par éclater entre ceux qui, regroupés autour de Pascal Affi N'Guessan, comprenaient - un peu tard que le boycott éternel serait contre-productif, et ceux qui, rassemblés autour d'Aboudramane Sangaré, persistaient à prôner le "Gbagbo ou rien ». Dans leur stratégie de la chaise vide, ces derniers bénéficiaient de la sortie fort opportune en librairie de trois ouvrages à charge contre Alassane Ouattara (Matteï, 2014; Pigeaud, 2015 et Houdin, 2015), sur lesquels ils s'appuyèrent pour continuer à contester les résultats de 2010.

En respectant le calendrier fixé par la CEI, on passa de 33 candidats déclarés à 14 candidats confirmés, puis à 10 candidats retenus par le Conseil constitutionnel, et enfin à 7 candidats menant campagne, puisque 3 " poids lourds $»^{11}$ avaient jeté l'éponge dans les derniers jours quand ils eurent conscience qu'ils risquaient l'humiliation dans les urnes. Le bulletin unique proposé aux électeurs contenait néanmoins dix noms.

23 La campagne électorale fut calme, mais disproportionnée dans les moyens déployés. Face à l'énorme machine du RHDP, seuls Pascal Affi N'Guessan (FPI) et KKB (dernier dissident du PDCI resté en course) sillonnèrent le pays pour tenir des meetings. Conformément à la 
loi, tous eurent accès aux médias d'État, mais les prestations s'avérèrent inégales et certains candidats ne purent cacher l'indigence de leurs propositions. Pour autant le climat politique semblait apaisé, ce qui rassurait les populations, comme si elles avaient oublié que la même période pré-électorale cinq ans plus tôt était tout aussi peu tendue. La journée du 25 octobre 2015 s'est déroulée sans incidents. Plusieurs centaines d'observateurs déployés dans le pays et surtout dans le gros district d'Abidjan (1 831467 électeurs, et 4472 bureaux de vote sur 19 849) n'ont rien relevé d'anormal, si ce n'est le dysfonctionnement des tablettes tactiles ${ }^{12}$ qui avaient été introduites dans le processus au dernier moment par la CEI, pour assurer le contrôle biométrique des électeurs et le transfert électronique des données.

Mais l'impression qui prévalait au soir de ce dimanche était le "manque d'engouement » des Ivoiriens pour ce scrutin pourtant important. La question de l'abstention revenait alors en boomerang, d'abord pour le camp présidentiel qui avait - bien imprudemment fait de la participation l'enjeu majeur de la consultation, et ensuite pour les dissidents du FPI qui avaient appelé au boycott et se préparaient à chiffrer l'impact de leur mot d'ordre et à le mettre au crédit de Gbagbo.

C'est donc sans doute par excès de zèle que la CEI annonça dès le lundi une participation «de l'ordre de $60 \%$ », alors que les chiffres de la plateforme de la société civile et des comptages parallèles ${ }^{13}$ ne franchissaient pas la barre des $53 \%$. De fait, le résultat officiel de la participation s'est stabilisé à 52,86 \%, soit trente points de moins qu'en 2010 (83,73\% au $1^{\text {er }}$ tour). La carte révèle toutefois une grande disparité selon les régions.

Illustration 1 - Participation par région

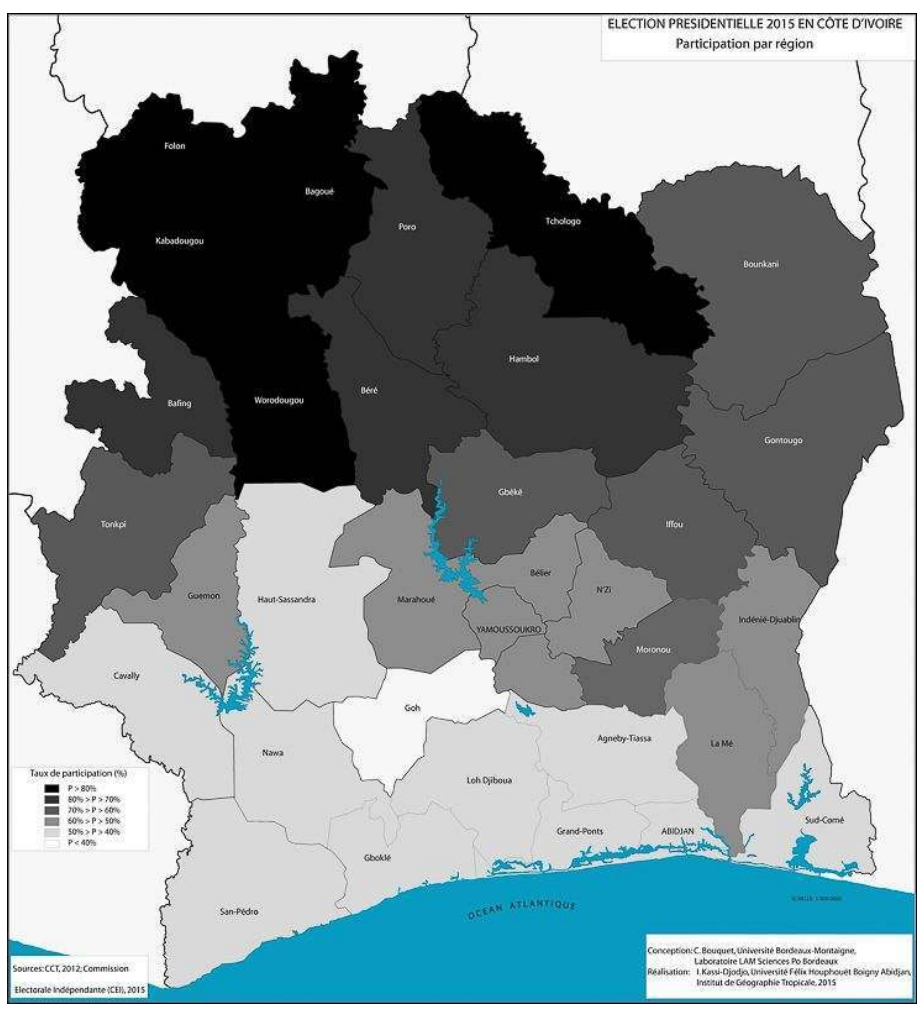

On retrouve les fortes mobilisations de 2010 dans les fiefs traditionnellement favorables à Ouattara: les régions de Séguéla, Odienné, Ferkessédougou, Boundiali, Korhogo, ont affiché une participation supérieure à $80 \%$. À l'inverse, le tiers sud-ouest du pays s'est 
assez peu mobilisé, et force est de constater qu'il s'agit des zones qui avaient soutenu Laurent Gbagbo. Plus surprenant: la participation dans le district d'Abidjan n'a pas dépassé $44 \%$, et elle a été faible dans les communes considérées comme acquises à Alassane Ouattara (Abobo : $48 \%$ ).

Les communicants proches du pouvoir ont tenté de fournir des explications, en arguant que l'élection de 2010 marquait une sortie de crise alors que celle de 2015 avait lieu dans un contexte apaisé et serein, où «l'évidence Ouattara » était telle que même ses propres électeurs pensaient la victoire acquise sans même avoir besoin de se déplacer... Du côté des inconditionnels de Gbagbo, certains se sont attribués les voix des 3 millions d'électeurs qui étaient restés chez eux. En fait, la lecture de la sociologie électorale de ce scrutin méritera une analyse bien plus approfondie que celles qui ont été livrées dans la presse et dans les cercles politiques de tous bords.

Une fois les résultats provisoires officiellement publiés (72 heures après la clôture), les commentateurs (et les courtisans) se sont focalisés sur l'ampleur du score de Ouattara exprimé en pourcentage des votants : 83,66\%. C'était sans surprise, compte tenu de la faiblesse de ses adversaires, puisque le candidat du FPI, Affi N'Guessan, n'a obtenu que 9,29\% et le dernier dissident du PDCI, Konan Kouadio Bertin, 3,88 \%. Le président sortant est arrivé en tête dans toutes les régions du pays, sauf dans le Moronou, région natale d'Affi.

Le dernier motif d'inquiétude concernant la paix civile fut levé lorsque tous les candidats eurent reconnu la victoire du président sortant, et que le Conseil constitutionnel eut confirmé les résultats provisoires.

31 Il ne restait plus qu'à analyser en profondeur les données chiffrées issues des urnes. Pour mieux tenir compte du poids réel qu'Alassane Ouattara représente régionalement, il a semblé pertinent de cartographier les pourcentages qu'il a obtenus par rapport aux inscrits plutôt que par rapport aux suffrages exprimés. Cela permet d'introduire l'abstention comme valeur de relativisation. Pour l'ensemble du pays, son score s'établit à $41,55 \%$ des inscrits. 
Illustration 2 - Pourcentage des suffrages obtenus par Ouattara par rapport aux inscrits (par région)

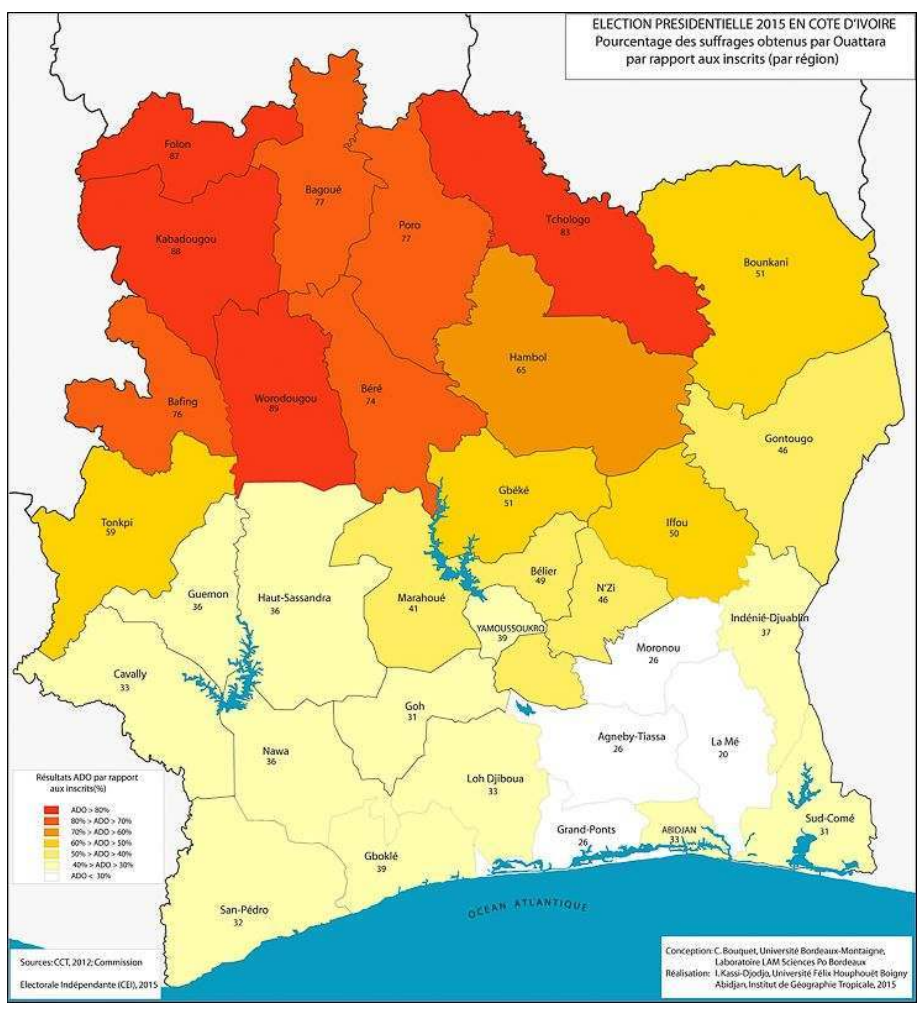

On retrouve à peu près les mêmes contours que sur la carte précédente. Le quart nordouest affiche des scores élevés en faveur du candidat du RHDP, mais un peu moins «soviétiques» que ceux qui découlent du comptage normal : $89 \%$ au lieu de 99 pour le Worodougou, $83 \%$ au lieu de 98 pour le Tchologo, $77 \%$ au lieu de 98 pour le Poro. Si l'on considère que la « légitimité absolue » correspond à $50 \%$ des inscrits, toute la moitié nord du pays a majoritairement soutenu Alassane Ouattara.

Mais on remarque que la moitié sud est restée beaucoup plus tiède, non seulement dans le tiers sud-ouest où la faible participation laissait entendre une faible adhésion, et où elle est comprise entre 30 et $40 \%$ des inscrits, mais aussi et surtout dans « l'écharpe blanche » qui entoure le district d'Abidjan, où quatre régions (Agnéby-Tiassa, Grands-Ponts, Moronou et La Mé) situent le score d'Alassane Ouattara sous la barre des $30 \%$ des inscrits. Ce bloc recouvre à peu près la zone où Laurent Gbagbo avait dépassé la barre des $50 \%$ des inscrits en 2010. L'ombre de l'ex-président ne s'est donc pas vraiment estompée dans le paysage politique ivoirien, même s'il est difficile de mesurer la densité de ses soutiens populaires à partir des chiffres de l'abstention. En tout état de cause, il n'y a pas d'adhésion forte à Ouattara dans cette partie du territoire.

En revanche, sur l'ensemble du pays, Alassane Ouattara a bel et bien obtenu davantage de suffrages sur son nom qu'au second tour de la présidentielle de 2010 (2 618229 voix contre 2483164 ), ce qui conforte l'idée qu'il a conservé son potentiel de popularité et d'adhésion.

Mais pas partout puisque dans le district d'Abidjan, là où les réalisations de son quinquennat étaient pourtant les plus visibles et spectaculaires, il a perdu près de 75000 voix, soit plus de $10 \%$ de son électorat. C'est un sujet de préoccupation pour son camp, 
car son score rapporté aux inscrits perd six points par rapport à $2010(33,4 \%$ contre $39,4 \%$ ), alors qu'il avait annoncé que, cette fois-ci, il gagnerait cette "bataille d'Abidjan » qu'il avait précédemment perdue face à Laurent Gbagbo (48,1 \% contre 51,9\%).

Illustration 3- Évolution des suffrages obtenus par Ouattara dans les communes du district d'Abidjan entre 2010 et 2015

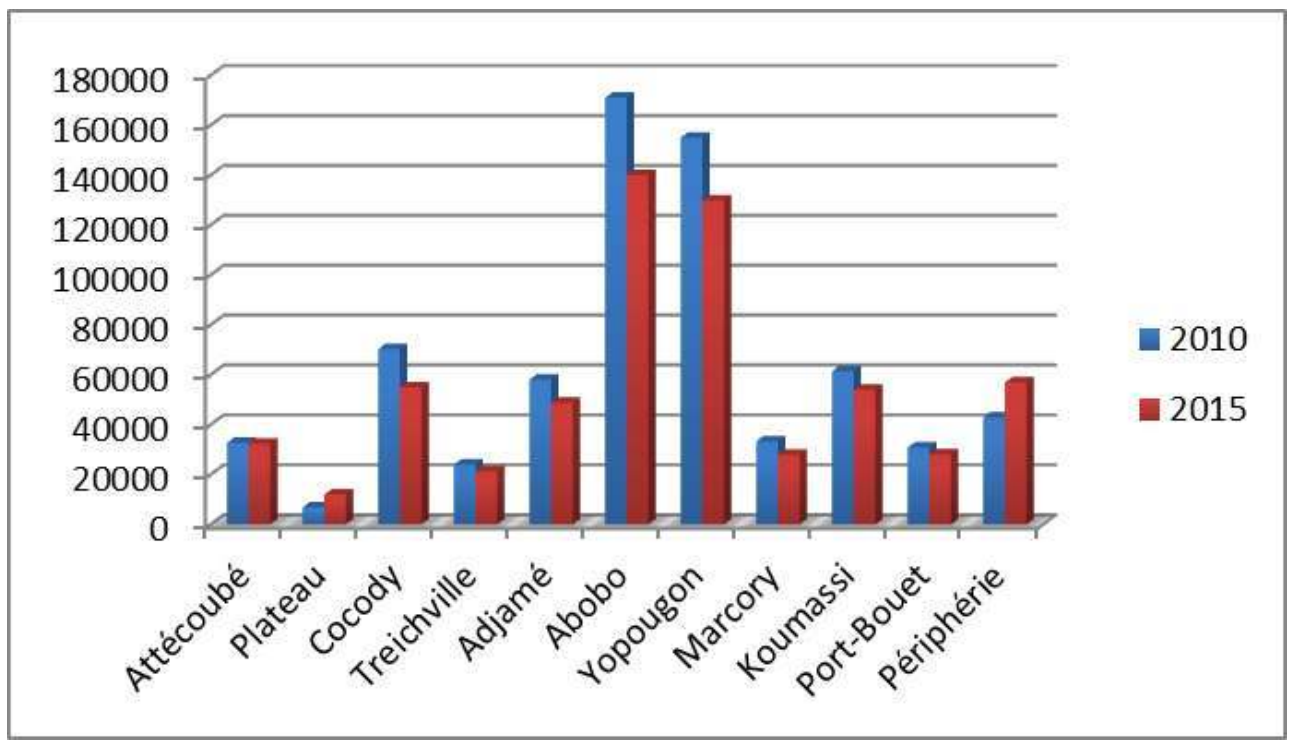

À l'exception des communes périphériques et de celle du Plateau (où le nombre des inscrits avait progressé de $50 \%$ ), Alassane Ouattara recule partout en valeur absolue, y compris dans son « fief » historique d'Abobo. Or ce n'est pas vraiment une surprise dans la mesure où les élections intermédiaires (les législatives de 2011 et les municipales de 2013) avaient déjà révélé une assez forte démobilisation dans le propre parti (RDR) du président. Au moment de l'enrôlement sur les listes électorales à partir de mars 2015, la sensibilisation avait également été faible dans les communes qui lui étaient favorables, et on a vu que la participation en octobre 2015 y avait été très modérée.

C'est une déception pour le camp présidentiel, qui devra étudier soigneusement les causes de cette désaffection, notamment du côté des électeurs des quartiers précaires, qui, dans le meilleur des cas, n'ont pas toujours ressenti les effets du changement promis et, au pire, ont été « déguerpis $»^{14}$ sans véritable solution alternative (Bouquet, Kassi-Djodjo, 2014-2).

Certes relatif et localisé, ce recul est néanmoins préoccupant dans la mesure où l'opposition n'a pas pesé lourd sur l'ensemble du pays. Avec 290780 voix, Affi n'a rassemblé que $14 \%$ de ce qu'avait obtenu Gbagbo au second tour de 2010 (2 107 055); il ne s'est donc pas imposé comme son successeur légitime. Avec 121386 voix, Konan Kouadio Bertin (KKB) n'a capitalisé que $10 \%$ des suffrages obtenus par Bédié au premier tour de 2010 (1 165 532) ; c'est trop peu pour espérer fonder une dissidence du PDCI.

Ainsi la victoire d'Alassane Ouattara n'est-elle pas le plébiscite que pourrait laisser supposer son score de $83 \%$, même si ses partisans ont parlé de triomphe. Cette forme de déni, qui avait déjà émergé après les élections intermédiaires où l'opposition était absente, devra rapidement être corrigée par la citation cornélienne (« à vaincre sans péril ... ») sous peine de conduire le parti au pouvoir vers une tentation hégémonique à risques. 


\section{Conclusion : la page est-elle vraiment tournée?}

Comme une grande majorité d'Ivoiriens, on retiendra d'abord les motifs de satisfaction: l'événement s'est déroulé sans tension, sans incidents, voire sans passion, et aucun candidat n'a été exclu. Les opérations de vote ont été transparentes et les résultats n'ont pas été contestés. Les vaincus ont tous reconnu la victoire d'Alassane Ouattara et ont assisté à sa prestation de serment. Autant d'indicateurs qui caractérisent une démocratie apaisée.

Une seconde lecture peut s'avérer plus critique, notamment si elle se focalise autour des deux cartes suivantes :

Illustration 4 - Comparaison des scores de Gbagbo et Ouattara en 2010 par rapport aux inscrits

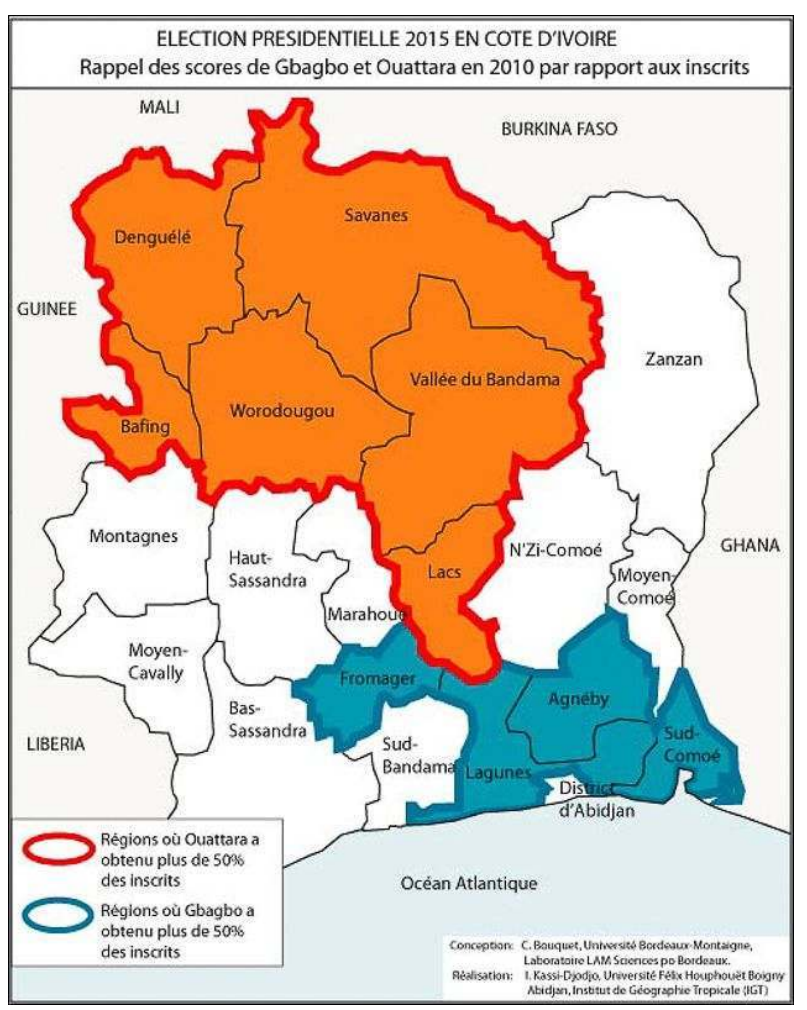


Illustration 5 - Comparaison des scores de Ouattara en 2015 et de Gbagbo en 2010 par rapport aux inscrits

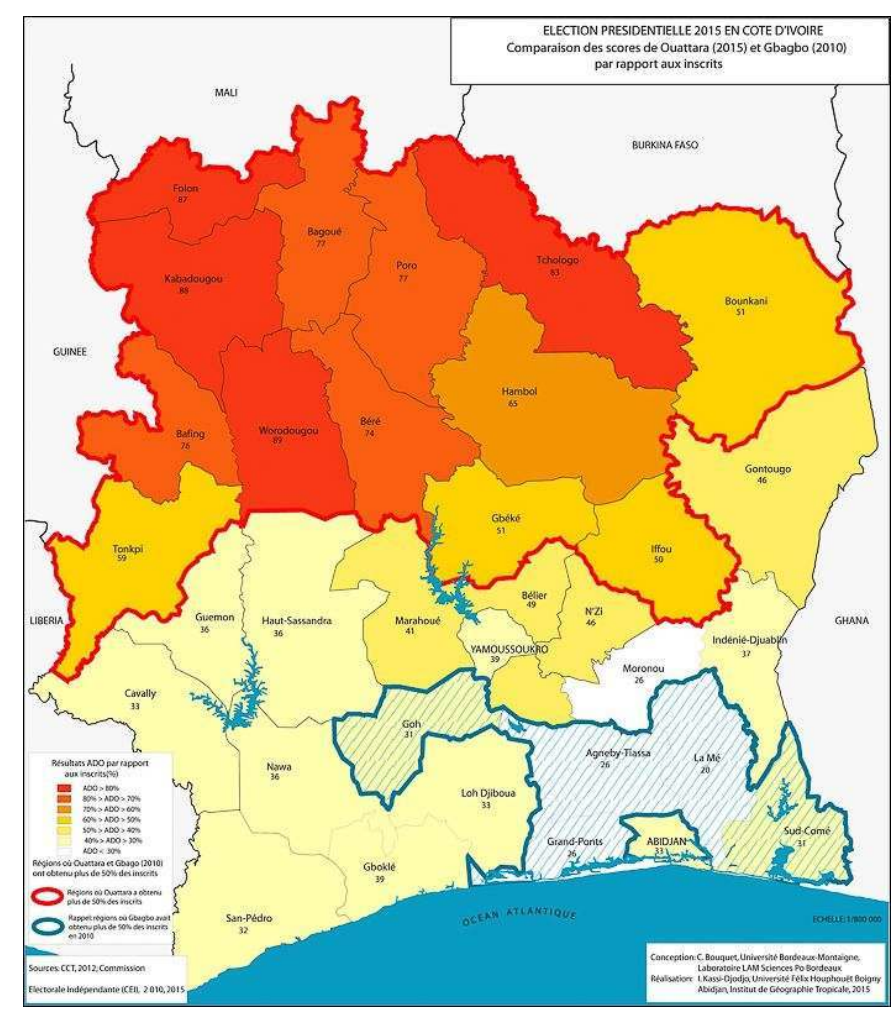

La première carte (illustration 4) a circonscrit les régions ${ }^{15}$ où chacun des deux candidats a obtenu, lors du second tour de l'élection présidentielle de 2010 , plus de $50 \%$ des inscrits. On remarque qu'ils se distinguent en deux blocs compacts et contigus, bien identifiés géographiquement.

L'illustration 5 a repris le bloc Gbagbo de 2010 - l'exploitation des données électorales semble nous y autoriser - et a circonscrit les régions où Ouattara a obtenu plus de $50 \%$ des inscrits lors de l'élection présidentielle de 2015. Le nouveau bloc Ouattara s'est légèrement élargi dans le grand-ouest (l'ex-région des Montagnes), dans le nord-est (la moitié nord de l'ex-région du Zanzan) et le centre-ouest (la moitié nord de l'ex-région du N'Zi-Comoë). Mais il s'est rétréci dans le centre, autour du district de Yamoussoukro, au point que les deux blocs ne sont plus contigus. Sur les deux cartes, aucune région entrant dans le critère retenu (plus de $50 \%$ des inscrits) n'est isolée par rapport aux blocs, qui restent donc compacts.

Le district d'Abidjan ne faisait pas partie du bloc Gbagbo en 2010, mais il y était majoritaire, et c'est là que se profile avec le plus d'acuité l'interrogation sous-jacente à ce montage cartographique: les positions de 2010 n'ont-elles pas eu tendance à se cristalliser? Derrière l'abstention relativement massive ne se cache-t-il pas une forme de radicalisation de l'électorat, dont une partie serait prête à suivre Laurent Gbagbo à son retour (?) ou le dauphin qu'il désignerait, simplement par rejet d'Alassane Ouattara? Les combats d'avril 2011, qui avaient plus particulièrement touché Yopougon et Cocody, n'ont-ils pas laissé dans les mémoires des traces indélébiles?

Autant dire que la page de 2010-2011 n'est pas réellement tournée. Certes, le temps va continuer à faire son œuvre d'oubli, mais le président réélu devra peut-être l'aider en 
s'attaquant à plusieurs problèmes qui sont restés en suspens, dont certains ont déjà été pointés à plusieurs reprises ${ }^{16}$ mais dont le principal reste celui de la réconciliation, qui passera par la justice pour tous.

Les prochaines échéances électorales seront l'occasion de vérifier si nos hypothèses sont recevables, et la future carte issue des législatives de 2016 apportera probablement des clarifications sur cette répartition par grands blocs.

\section{BIBLIOGRAPHY}

Akindes F., 2012. Côte d'Ivoire : pardonner ou punir? Alternatives Internationales, Hors-série $\mathrm{n}^{\circ} 10$, p. 94-95.

Bouquet C., 2011. Côte d'Ivoire. Le désespoir de Kourouma. A. Colin, 333 p.

Bouquet C., Kassi-Djodjo I., 2012. Les élections législatives en Côte d'Ivoire marquent-elles la sortie de crise ? EchoGéo [En ligne], Sur le Vif, mis en ligne le 26 mars 2012. URL : http:// echogeo.revues.org/13017 ; DOI : 10.4000/echogeo.13017

Bouquet C., Kassi-Djodjo I., 2014-1. Les élections locales 2013 en Côte d'Ivoire : des malentendus subsistent sur l'exercice de la démocratie. EchoGéo [En ligne], Sur le Vif, mis en ligne le 13 janvier 2014. URL : http://echogeo.revues.org/13697 ; DOI : 10.4000/echogeo.13697

Bouquet C., Kassi-Djodjo I., 2014-2. Déguerpir' pour reconquérir l'espace public à Abidjan. L'Espace Politique, $\mathrm{n}^{\circ} 22$ (2014-1), 16 p. http://espacepolitique.revues.org/2963

Choi Y.J., 2015. La crise ivoirienne. Ce qu'il fallait comprendre. Michel Lafon, 299 p.

Dozon J.-P., 2011. Les clefs de la crise ivoirienne. Karthala, 144 p.

Houdin B., 2015. Les Ouattara. Une imposture ivoirienne. Éditions du moment, 334 p.

Hofnung T., 2011. La crise ivoirienne. De Félix Houphouët-Boigny à la chute de Laurent Gbagbo. La Découverte, $191 \mathrm{p}$.

Koné G., 2011. Logiques sociales et politiques des pillages et barrages dans la crise post-électorale en Côte d'Ivoire. Politique africaine, $\mathrm{n}^{\circ} 122$, p. 145-160.

Matteï F., Gbagbo L., 2014. Pour la vérité et la justice. Éditions du moment, 320 p.

Miran M., 2015. Guerres mystiques en Côte d'Ivoire. Religion, patriotisme, violence (2002-2013).

Karthala, 372 p.

Miran M., 2011. Au-delà du silence et de la fureur. Duékoué (Ouest ivoirien) : rencontres interreligieuses au 'carrefour de la haine. Politique Africaine, n 123, p. 95-115.

Nkenazi Zena P., 2013. Leçons et limites du DDR en Afrique. Bulletin de la Sécurité Africaine (CESA), $\mathrm{n}$ $\circ 24,8 \mathrm{p}$.

Notin J.-C., 2013. Le crocodile et le scorpion. La France et la Côte d'Ivoire (1999-2013). Éditions du Rocher, $441 \mathrm{p}$.

Pigeaud F., 2015. France Côte d'Ivoire. Une histoire tronquée. Vents d'ailleurs, 349 p. 


\section{NOTES}

1. Il convient de noter que l'ex-président Bédié, responsable du PDCI (Parti démocratique de Côte d'Ivoire), avait signé en 2005 un accord avec son adversaire d'autrefois, Alassane Ouattara, leader du RDR (Rassemblement des Républicains), et que ses engagements avaient été tenus puisque Alassane Ouattara avait été élu au second tour de 2010 grâce aux $25 \%$ de suffrages émanant des rangs de l'ancien parti unique. Cette alliance porte le nom de RHDP (Rassemblement des Houphouëtistes pour le Développement et la Paix).

2. Depuis 1990, on dénombre 23 opérations de DDR (Désarmement, Démobilisation, Réinsertion) sur le continent africain (Nkénazi Zéna, 2013).

3. Crimes contre l'humanité, meurtres, viols, actes inhumains et persécutions.

4. La Côte d'Ivoire a refusé de transférer Simone Gbagbo à la CPI, préférant la juger sur place. À l'issue d'un procès approximatif, elle a été condamnée à 20 ans de prison par le tribunal d'Abidjan le 9 mars 2015. http://lamenparle.hypotheses.org/221

5. Attention : les deux articles d'ÉchoGéo qui couvrent ces scrutins intermédiaires sont illustrés par des cartes dans lesquelles les couleurs des partis sont obsolètes, puisque la charte graphique du RDR impose désormais la couleur orange, au lieu du bleu que nous avions utilisé dans toutes nos cartes électorales depuis 2000 en nous basant sur l'idéologie libérale du parti de Ouattara.

6. Discours prononcé lors de son séjour à Abidjan le 07/01/2013 https://www.imf.org/external/ french/np/speeches/2013/010713f.htm

7. http://lamenparle.hypotheses.org/20\#more-20

8. Les résultats complets du RGPH 2014 (Recensement Général de la Population et de l'Habitat) n'ont toujours pas été publiés, de telle sorte que des doutes sérieux sont apparus concernant sa fiabilité. (NB : il est très fréquent qu'il y ait des décalages importants entre les recensements et leur publication en Afrique ; le lien avec la question de leur fiabilité n'est pas automatique...)

9. À titre de comparaison, le Ghana voisin comptait près de 14 millions d'électeurs en 2012, et plus de 11 millions se sont rendus aux urnes pour l'élection présidentielle de décembre 2012 (pour une population de 26 millions d'habitants).

10. Il est âgé de 47 ans, mais dans la culture encore gérontocratique du PDCI, il est considéré comme « jeune ».

11. Une Coalition Nationale pour le Changement ( $\mathrm{CNC}$ ) avait été mise en place pour tenter de peser face au RHDP. Elle comprenait les trois dissidents du PDCI, dont Charles Konan Banny et Amara Essy, qui se retirèrent quelques jours avant le vote, ainsi que Mamadou Koulibaly, ultralibéral transfuge du FPI (à tendance socialiste...), qui adopta la position paradoxale du candidat qui n'est plus candidat mais qui bat campagne pour l'abstention.

12. Fournies par la société Morpho (ex-Safran) pour un montant voisin de 2 millions de dollars, ces tablettes étaient correctement programmées pour les 19849 bureaux de vote, mais elles n'avaient pas toujours été distribuées au bon endroit et les 60000 agents déployés n'avaient reçu que deux heures de formation avant de les manipuler.

13. Émanation du NDI (National Democratic Institute), la POECI (Plateforme d'Observation des Élections en Côte d'Ivoire) regroupait 14 organisations de la société civile. Elle avait mis au point un mode de comptage par sondage qui, recoupé avec les comptages parallèles par smartphones des appareils politiques, avait rapidement donné les tendances, puis des résultats précis que la CEI ne pouvait pas contester.

14. En Afrique subsaharienne, les «déguerpissements » sont des opérations qui consistent à déplacer, souvent sans ménagement, les habitants des quartiers précaires. Les victimes sont couramment appelées des « déguerpis ». 
15. La carte administrative des régions a été largement modifiée en 2011, mais on parvient à s'y retrouver sur la base des départements, qui ont pu être redistribués d'une région à l'autre, mais dont les limites n'ont pas bougé.

16. https://lamenparle.hypotheses.org/380

\section{ABSTRACTS}

The presidential election recently held in Côte d'Ivoire was peaceful and, unlike the previous poll in 2010, its results have not been disputed. Facing a weakened and divided opposition, Alassane Ouattara won with $83.66 \%$ of the votes cast. However, the turnout was relatively low $(52.86 \%)$ and his score out of the 6301189 registered voters was only $41.55 \%$.

In absolute value, the outgoing president gained the same total amount of votes compared to 2010 while regressing in Abidjan, including his traditional strongholds (Abobo, Adjame), despite new and remarkable infrastructures benefitting the megacity which might have prompted a more robust adhesion from voters.

L'élection présidentielle qui a eu lieu en Côte d'Ivoire s'est déroulée dans le calme et, contrairement au scrutin précédent de 2010, ses résultats n'ont pas été contestés. Face à une opposition affaiblie par les divisions, Alassane Ouattara l'a emporté avec 83,66\% des suffrages exprimés. Mais la participation a été relativement faible $(52,86 \%)$, et son score par rapport aux 6301189 inscrits n'est que de 41,55\%.

En valeur absolue, le président sortant retrouve son volume de voix de 2010. Mais il recule à Abidjan, y compris dans ses fiefs traditionnels (Abobo, Adjamé), alors que la mégapole a bénéficié d'infrastructures nouvelles spectaculaires qui auraient pu emporter une adhésion plus massive des électeurs.

\section{AUTHORS}

\section{CHRISTIAN BOUQUET}

Christian Bouquet, Christian.Bouquet@u-bordeaux-montaigne.fr, est Professeur émérite à l'université de Bordeaux-Montaigne, chercheur à LAM (Les Afriques dans le Monde), Sciences Po Bordeaux.

\section{IRÈNE KASSI-DJODJO}

Irène Kassi-djodjo, irenekassi@yahoo.fr, est Maître-Assistant à l'Université Félix HouphouëtBoigny d'Abidjan, Institut de géographie tropicale. 